\title{
A Conceptual Spectral Plume Model for Understanding Tropical Temperature Profile and Convective Updraft Velocities
}

\author{
Wenyu Zhou And Shang-PING XIE \\ Scripps Institute of Oceanography, University of California, San Diego, La Jolla, California
}

(Manuscript received 14 November 2018, in final form 15 May 2019)

\begin{abstract}
The tropical tropospheric temperature is close to but typically cooler than that of the moist adiabat. The negative temperature deviation from the moist adiabat manifests a $\mathrm{C}$-shape profile and is projected to increase and stretch upward under warming in both comprehensive climate models and idealized radiativeconvective equilibrium (RCE) simulations. The increased temperature deviation corresponds to a larger convective available potential energy (CAPE) under warming. The extreme convective updraft velocity in RCE increases correspondingly but at a smaller fractional rate than that of CAPE. A conceptual model for the tropical temperature deviation and convective updraft velocities is formulated to understand these features. The model builds on the previous zero-buoyancy model but replaces the bulk zero-buoyancy plume by a spectrum of entraining plumes that have distinct entrainment rates and are positively buoyant until their levels of neutral buoyancy. Besides the negative temperature deviation and its increasing magnitude with warming, this allows the spectral plume model to further predict the C-shape profile as well as its upward stretch with warming. By representing extreme convective updrafts as weakly entraining plumes, the model is able to reproduce the smaller fractional increase in convective velocities with warming as compared to that of CAPE. The smaller fractional increase is mainly caused by the upward stretch in the temperature deviation profile with warming, which reduces the ratio between the integrated plume buoyancy and CAPE. The model thus provides a useful tool for understanding the tropical temperature profile and convective updraft velocities.
\end{abstract}

\section{Introduction}

To first order, the tropospheric temperature profile in the tropics can be thought of as following the moist adiabat, that is, an undiluted updraft from the surface (e.g., Riehl and Malkus 1958; Xu and Emanuel 1989). This has been a useful conceptual model for understanding many important tropical phenomena, such as reduced atmospheric stratification compared to the dry adiabat and enhanced warming in the upper troposphere compared to the surface under global warming. A closer look, however, shows that the tropical atmosphere is generally cooler than the moist adiabat. The negative temperature deviation from the moist adiabat features a C-shape profile with the peak located in the middle to upper troposphere. Thus, the convective available potential energy (CAPE), defined as the integrated buoyancy of the moist adiabat parcel, is typically positive in the tropics and determined by the

Corresponding author: Wenyu Zhou, zhouwy1128@gmail.com magnitude of this negative deviation. Under global warming, the negative temperature deviation and the consequent CAPE are projected to increase in global climate models (GCMs; e.g., Sobel and Camargo 2011; Fasullo 2012), implying stronger convective updraft velocities (Singh and O'Gorman 2015) and more severe thunderstorms, lightning, and precipitation in the future (e.g., Williams et al. 1992; Brooks 2013; Lepore et al. 2015; Romps et al. 2014; Singh et al. 2017). Another important change with warming that has not been emphasized in previous studies is the upward shift in the relative height of the peak temperature deviation in the troposphere, which could affect the vertical profile of the cloud buoyancy and velocities. It is thus of great importance to understand why the cold deviation arises, how it changes with global warming, and what it implies for convective velocities.

Radiative-convective equilibrium (RCE), in which the atmosphere is in a pure balance between the radiative and convective processes, is a useful idealized framework for understanding the tropical atmosphere (e.g., Manabe and Wetherald 1967; Tompkins and Craig 1998; 
Held et al. 1993; Bretherton et al. 2005). Both the negative $\mathrm{C}$-shape deviation and its changes with warming are consistently simulated in RCE (e.g., Romps 2011; Muller et al. 2011; Singh and O'Gorman 2013; Seeley and Romps 2015). Since no large-scale circulations are included in $\mathrm{RCE}$, this indicates that the aforementioned features may be explained from moist thermodynamics without considering interactions with large-scale circulations. Furthermore, cloud-resolving RCE simulations provide a simple framework to study the response of small-scale convective updrafts to warming. It is found that the extreme convective updraft velocity increases correspondingly with CAPE under warming but the fractional increasing rate is smaller than that of CAPE (Singh and O'Gorman 2015).

The success of RCE has motivated studies to formulate simpler conceptual models to further understand the underlying physics. An important process neglected in the undiluted moist adiabat picture is the entrainment effect on convective updrafts. Both observations (e.g., Stommel 1947; Taylor and Baker 1991) and RCE simulations (e.g., Lin and Arakawa 1997; Romps and Kuang 2010a; Pauluis and Mrowiec 2013) confirm that convective updrafts are highly diluted by entrainment from the environment and undiluted updrafts are rare or virtually absent in the upper troposphere outside of tropical cyclones (Romps and Kuang 2010b). By simplifying the convective clouds into a bulk-entraining plume and assuming that the environment is neutrally buoyant to this bulk-entraining plume, Singh and O'Gorman (2013) build a conceptual model of RCE. The model is able to explain the negative temperature deviation as well as its increasing magnitude with warming. It explains that the amplified saturation deficit in the free troposphere with warming leads to larger entrainment dilution of moist static energy (MSE) in clouds and consequently results in larger negative deviation of the environmental temperature from the moist adiabat. The model however fails to reproduce the $\mathrm{C}$-shape profile of the temperature deviation as well as its upward stretch under global warming. This induces biases in the buoyancy profile of convective clouds and the estimated convective updraft velocities.

To provide a more satisfactory understanding of the deviation profile and its implication on convective updraft velocities, here, we formulate a more realistic conceptual model of RCE by dropping the zerobuoyancy assumption and representing the convective clouds by a spectrum of entraining plumes instead of a bulk, zero-buoyancy plume. We show that the spectral plume model is able to explain the $\mathrm{C}$-shape profile of the negative temperature deviation as well as its increasing magnitude and upward stretch with warming. Despite being more sophisticated by design, the model has a concise analytical formula that reveals an important term that has been implicitly ignored by the zero-buoyancy plume model but turns out to be essential for reproducing the C-shape deviation. Furthermore, the model successfully reproduces the smaller fractional increase of the convective updraft velocity with warming compared to that of CAPE. It suggests that the reduced increase is mainly caused by the upward stretch of the temperature deviation with warming and additionally contributed from the positive cloud-base energy anomaly associated with the extreme convective updrafts.

The paper is organized as follows. The datasets and methods are described in section 2. In section 3, we present the tropical temperature profiles in observations and simulations of the GCM and RCE. After briefly reviewing the zero-buoyancy plume model in section 4 , we formulate the more sophisticated spectral plume model in section 5 . In section 6 , we use the spectral plume model to understand the response of convective updraft velocities to warming. Section 7 is a summary, and section 8 is a discussion.

\section{Datasets and methods}

\section{a. Tropical sounding}

The tropical moist (MT) sounding from Dunion (2011) is used as a representative temperature profile over tropical convecting regions. The MT sounding is an updated version of the Jordan hurricane season sounding (Jordan 1958) by distinguishing air masses with different thermodynamic and kinematic characteristics from about 6000 rawinsonde observations over the Caribbean Sea region during the 1995-2002 hurricane seasons (July-October).

\section{b. GCM simulations}

Climate simulations of the Last Glacial Maximum (last 50 years of the simulation), the historical (1979-2005), and representative concentration pathways (RCP) 8.5 (2080-2100) using the CCSM4 GCM (Gent et al. 2011) from the CMIP5 (Taylor et al. 2012) archive are used to represent the cold, normal, and warm climates, respectively. The tropical-mean temperature profiles are computed as the average from $10^{\circ} \mathrm{S}$ to $10^{\circ} \mathrm{N}$. The tropical mean surface temperature at these three climates are 297.3, 300.4, and $303.2 \mathrm{~K}$, respectively.

\section{c. RCE simulations}

RCE simulations are conducted in a doubly periodic domain using the fully compressible and nonhydrostatic model (CM1; Bryan and Fritsch 2002). The bottom boundary condition is a saturated surface with prescribed homogeneous surface temperature. Surface fluxes 
and stresses are parameterized based on the MoninObukhov theory. The microphysics scheme is a singlemoment scheme based on Lin et al. (1983). The subgrid turbulence effect is parameterized by the Smagorinsky scheme. The NASA Goddard radiative transfer scheme (radopt $=1$ ) is used. The solar flux is fixed at $390 \mathrm{~W} \mathrm{~m}^{-2}$ with a constant zenith angle of $43^{\circ}$. The $\mathrm{CO}_{2}$ concentration in the atmosphere is fixed at $300 \mathrm{ppm}$. The model uses a horizontal resolution of $2 \mathrm{~km}$ and includes $48 \mathrm{un}$ evenly spaced vertical levels with the model top at $22 \mathrm{~km}$. Rayleigh damping is applied above $18 \mathrm{~km}$ to prevent gravity wave reflection. The size of the doubly periodic domain is $96 \mathrm{~km} \times 96 \mathrm{~km}$. Each simulation runs for 120 days, and hourly outputs from the last 20 days are collected for analysis. To investigate the effect of surface warming, simulations are conducted at various surface temperatures from 291 to $311 \mathrm{~K}$.

\section{d. Inputs for conceptual models}

Near-surface air temperature and specific humidity from the RCE simulations are used as the surface condition for the conceptual models. A constant relative humidity of $70 \%$ is assumed for the free troposphere above the cloud base. For the purpose of simplicity, we assume that all cloud water precipitates immediately.

For the zero-buoyancy plume model, the following vertically varying entrainment rate $\varepsilon$ is assumed for the bulk-entraining plume to account for the decreasing entrainment rate with the height $z$ :

$$
\varepsilon[z]=\left\{\begin{array}{cc}
\varepsilon_{o}\left(\frac{z_{t}-z}{z_{t}-z_{b}}\right)^{k}, & z_{b}<z<z_{t} \\
0, & \text { elsewhere }
\end{array}\right.
$$

where $z_{t}$ is the tropopause height and $z_{b}$ is the cloudbase height (defined as the level where the plume first becomes saturated). We use $\varepsilon_{o}=0.3 \mathrm{~km}^{-1}$ and $k=1$ to best fit the RCE simulations. We assume that the vertical profile of the entrainment rate does not change substantially with warming so that its change with warming plays a secondary role in affecting the temperature deviation change.

For the spectral plume model, each plume has a distinct entrainment rate that does not vary with height. In this model, plumes with weak entrainment rates would reach neutral buoyancy at higher levels and a one-to-one relationship between the plume's entrainment rate $\varepsilon$ and the plume's level of neutral buoyancy $z_{d}$ is assumed as follows:

$$
\varepsilon\left[z_{d}\right]=\left\{\begin{array}{cc}
\varepsilon_{o}\left(\frac{z_{t}-z_{d}}{z_{t}-z_{b}}\right)^{k}, & z_{b}<z<z_{t} \\
0, & \text { elsewhere }
\end{array}\right.
$$

We use $\varepsilon_{o}=0.45 \mathrm{~km}^{-1}$ and $k=1$ to best fit the RCE simulations. We assume that this relationship does not change substantially with warming so that its change with warming plays a secondary role in affecting the temperature deviation change. The model parameter sensitivity is discussed in section 8 .

The tropopause height $z_{t}$ is estimated using the surface MSE. The tropopause, which separates the troposphere and the stratosphere, can be seen as the highest level that moist convection could reach. The neutral buoyancy of the undiluted plume at the tropopause means the troposphere temperature is related to the surface MSE as

$$
T\left(z_{t}\right)=h_{b}-\frac{g}{C_{p}} z_{t}
$$

where $g$ is the gravitational constant, $C_{p}$ is the isobaric specific heat capacity of air, and $h_{b}$ is the surface MSE in the unit of temperature (divided by $C_{p}$ ). By assuming the stratosphere to be transparent to solar radiation and gray to longwave radiation, the stratosphere temperature can be formulated as (Held 1982)

$$
T(z)=\left\{\frac{I}{\sigma}\left[\frac{1}{2}+\frac{3}{4} \tau(z)\right]\right\}^{1 / 4}, \quad z \geq z_{t},
$$

where $I$ is the outgoing longwave radiation at the top of the atmosphere, $\tau(z)$ is the optical depth at level $z$, and $\sigma$ is the Stefan-Boltzmann constant. To satisfy the continuity in temperature at the tropopause, one has

$$
z_{t}=\frac{C_{p}}{g}\left(h_{b}-0.84 T_{e}\right) .
$$

Here, we have neglected the small $\tau\left(z_{t}\right)$ so that tropopause temperature is invariant and $T_{e}=(I / \sigma)^{1 / 4}=255 \mathrm{~K}$ is the effective emitting temperature.

\section{e. Computation of CAPE}

CAPE is computed as the accumulated buoyant energy of the undiluted plume from the level of free convection to the level of neutral buoyancy. The MSE of the undiluted plume is computed as the mean from the surface to $500 \mathrm{~m}$.

\section{Tropical profiles in observations and simulations}

Figure 1a shows the deviation from the moist adiabat of the environmental air temperature and MSE observed over the tropical ocean. The environmental saturation MSE is defined as

$$
\bar{h}^{*}=C_{p} \bar{T}+L_{v} \overline{q_{v}^{*}}+g z
$$



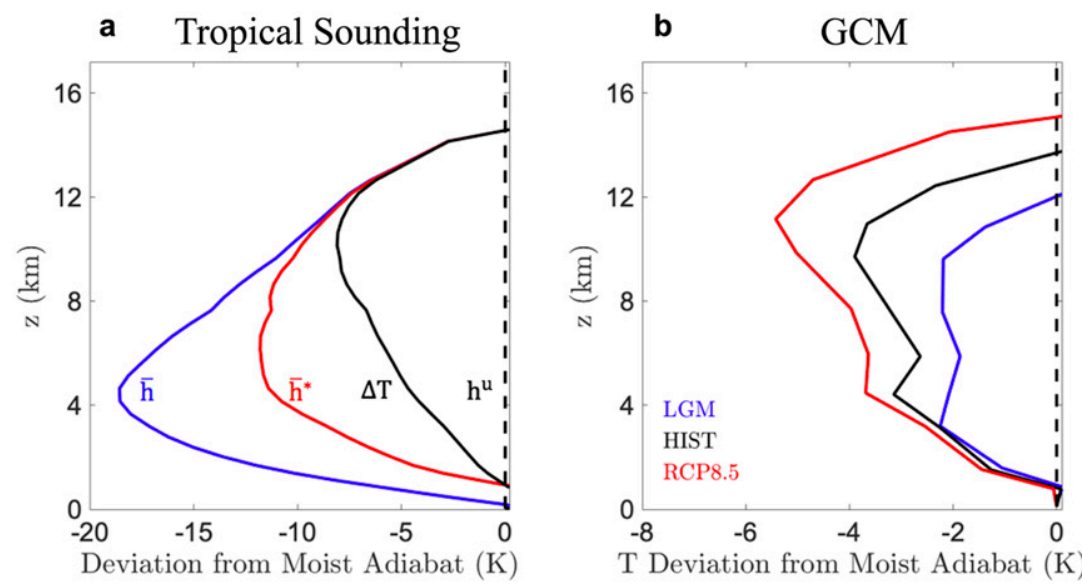

$\mathrm{T}$ Deviation from Moist Adiabat (K)
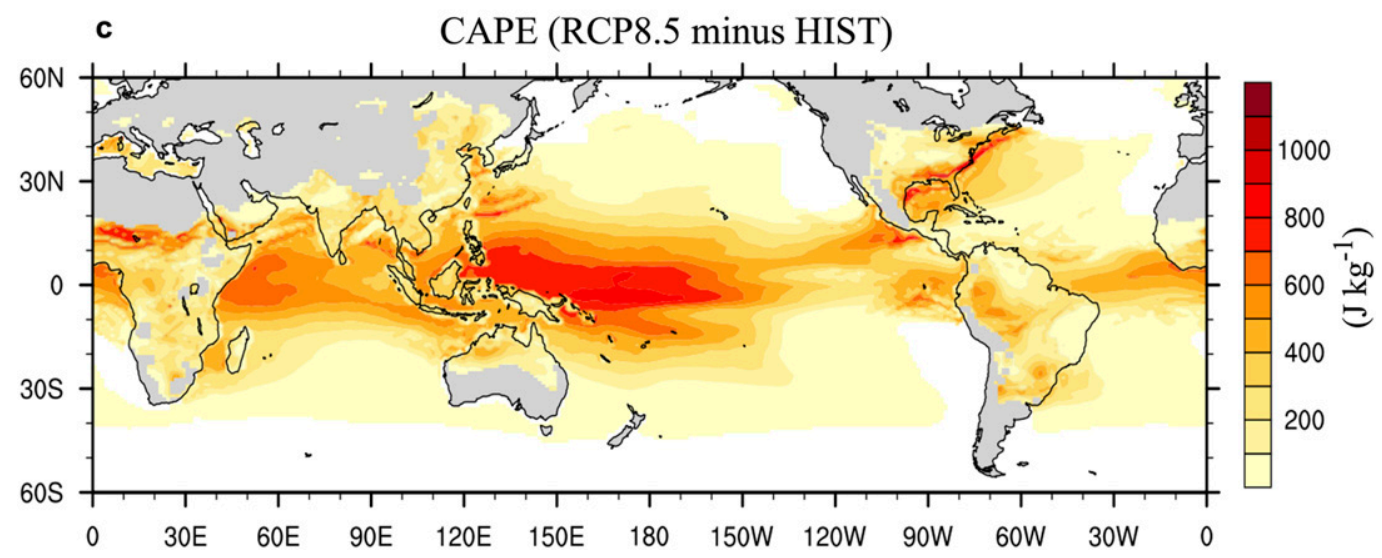

FIG. 1. (a) Vertical profiles of the environmental air temperature (black), MSE (blue) and saturation MSE (red) relative to the undiluted moist adiabat (dashed) observed in the tropical oceans. (b) Vertical profiles of the tropical temperature deviations simulated in GCMs under cold, normal, and warm climates, i.e., the Last Glacial Maximum (LGM, blue), the historical (1979-2005) period (HIST, black) and the RCP8.5 (2080-2100) scenario (red).

(c) Changes in the annual-mean CAPE from HIST to RCP8.5.

where $\bar{T}$ and $\overline{q_{v}^{*}}$ are the environmental temperature and saturation specific humidity, $z$ is the height above the surface, $C_{p}$ is the isobaric specific heat capacity of air, $L_{v}$ is the latent heat of vaporization, and the overbar symbol indicates the mean environment. The MSE of the undiluted moist adiabat is conserved so that

$$
h^{u}=C_{p} T^{u}+L_{v} q_{v}^{u}+g z=\text { const. }
$$

where $T^{u}$ and $q_{v}^{u}$ are the temperature and saturation specific humidity of the undiluted plume. Please note that our definition of MSE is simplified by neglecting the effect of phase changes between liquid and solid water. According to the linearization of the Clausius-Clapeyron relation, the temperature deviation from the moist adiabat $\Delta T=\bar{T}-T^{u}$ is related to the MSE deviation $\Delta h=\bar{h}^{*}-h^{u}$ as

$$
\Delta T=\frac{1}{1+\frac{L_{v}}{R_{v} \bar{T}^{2}} \frac{L_{v} \overline{q_{v}^{*}}}{C_{p}}} \Delta h .
$$

Both the temperature deviation $\Delta T$ (black) and the MSE deviation $\Delta h$ (red) are negative and manifest C-shape profiles with the peak deviations in the middle to upper troposphere (Fig. 1a). Because of the decreasing $\overline{q_{v}^{*}}$ with height in the denominator of (8), the height of the peak $\Delta T$ is higher than that of the peak $\Delta h$.

The negative deviation from the moist adiabat is projected to increase with global warming in GCMs (e.g., Sobel and Comargo 2011; Fasullo 2012). Figure 1b shows the tropical temperature deviation simulated by the CCSM4 GCM in the cold, normal, and warm climates (see section $2 b$ for details). The temperature deviation increases rapidly with warming (Fig. 1b), leading to increasing CAPE in the tropics (Fig. 1c). Idealized RCE simulations successfully reproduce the C-shape profile of the negative deviation (Fig. 2a) and the increasing magnitude with warming (Fig. 2b). Another important feature that is consistently simulated in both the GCM and RCE is that the relative height of the peak 
temperature deviation (to the tropopause height that increases itself) in the troposphere shifts upward with warming, manifesting an upward stretch of the C-shape profile. This change turns out to be important for explaining the changes in the convective updraft velocity with warming.

Since no large-scale circulations are included in the idealized RCE simulations, the success of the RCE simulations in reproducing GCM results suggests that the $\mathrm{C}$-shape temperature deviation and its changes with warming may be understood from moist thermodynamics without considering interactions with largescale circulations. This motivates studies to formulate simpler conceptual models of RCE to further explore the underlying physics.

\section{Zero-buoyancy plume model}

Inspired by the small cloud buoyancy relative to that of the undiluted plume (Muller et al. 2011; Romps and Öktem 2015), Singh and O'Gorman (2013) formulated a simple conceptual model of RCE in which the atmosphere is assumed to be neutrally buoyant with respect to a bulk-entraining plume. The model is able to reproduce the increasing negative deviation with warming. Here, we briefly review its formulation and limitations as a prerequisite for the more sophisticated spectral plume model.

By ignoring virtual temperature effects, the zerobuoyancy assumption implies the same temperature and consequently the same saturation MSE between the environment and the entraining plume; that is,

$$
\bar{h}^{*}=h^{\varepsilon},
$$

where $h^{\varepsilon}$ is the MSE of the entraining plume.

The plume MSE decreases with height because of the entrainment of the unsaturated environmental air; that is,

$$
\frac{d h^{\varepsilon}}{d z}=-\varepsilon[z]\left(h^{\varepsilon}-\bar{h}\right),
$$

where $\varepsilon[z]$ is the entrainment rate of the bulk plume at level $z$ and $\bar{h}$ is the environmental MSE.

Considering the conserved MSE of the undiluted plume, (9) and (10) can be combined into an equation for solving the MSE deviation $\Delta h=\bar{h}^{*}-h^{u}$ as

$$
\frac{d \Delta h}{d z}=-\varepsilon[z]\left(\bar{h}^{*}-\bar{h}\right)=-\varepsilon[z](1-\mathrm{RH}) \frac{L_{v} \overline{q_{v}^{*}}}{C_{p}},
$$

where RH is the environmental relative humidity.
One can then integrate (11) upward from the surface to get the vertical profile of $\Delta h$ and consequently $\Delta T$ according to (8) as

$$
\Delta T(z)=-\frac{1}{1+\frac{L_{v}}{R_{v} \bar{T}^{2}} \frac{L_{v} \overline{q_{v}^{*}}}{C_{p}}} \int_{z_{b}}^{z} \varepsilon[z](1-\mathrm{RH}) \frac{L_{v} \overline{q_{v}^{*}}}{C_{p}} d z .
$$

The predicted $\Delta h$ using surface temperature and specific humidity from the control RCE simulation is shown in Fig. $2 \mathrm{c}$ (see section $2 \mathrm{~d}$ for details) and the predicted $\Delta T$ for different surface temperatures are plotted against the results from the RCE simulations in Fig. 2d. The increasing negative temperature deviation with warming is reproduced by the zero-buoyancy plume model. This can be analytically understood from (12). As the saturation specific humidity $\overline{q_{v}^{*}}$ increases with warming, the fractional increase in the integrand dominates the fractional increase in the denominator, leading to increased negative temperature deviation $\Delta T$. Physically, the zero-buoyancy plume illustrates that the increased saturation deficit with warming leads to stronger MSE dilution from entrainment. The environmental temperature, which is identical to the plume temperature under the zero-buoyancy assumption, then deviates further away from the moist adiabat.

However, the above explanation relies on the assumption of zero cloud buoyancy, which could be systematically biased as clouds are in general positively buoyant. As shown in Fig. 2d, the model fails to reproduce the C-shape profile of the deviation that is consistently seen in both observations and simulations. By considering the environment to be neutrally buoyant to a bulk-entraining plume whose MSE decreases monotonically with height, (11) predicts $\Delta h$ to increase monotonically in magnitude with height and reach a maximum at the tropopause. In reality, convective clouds consist of a wide spectrum of entraining plumes with distinct entrainment properties and they would reach neutral buoyancy at different levels of the troposphere. Condensing such a plume spectrum into a bulk-entraining plume with zero buoyancy at all levels inevitably induces biases in the predicted temperature profile. In particular, since the plumes reaching the upper (lower) troposphere would generally have a smaller (larger) entrainment rate than the bulk-averaged plume, there would be warm bias in the lower troposphere and cold bias in the upper troposphere (Fig. 2d).

\section{Spectral plume model}

Here, we seek to provide a more satisfactory understanding of the C-shape temperature deviation, its 


\section{RCE Simulations}

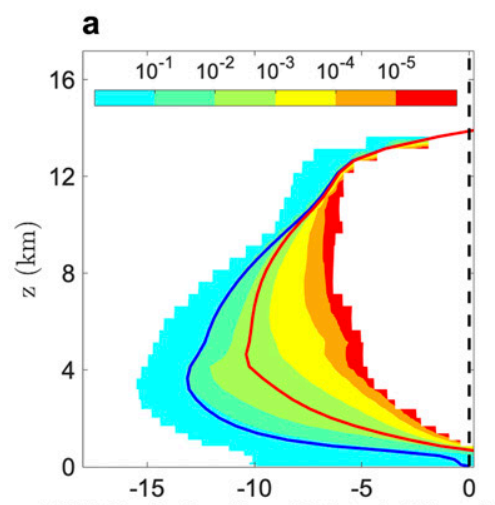

MSE Deviation from Moist Adiabat (K)

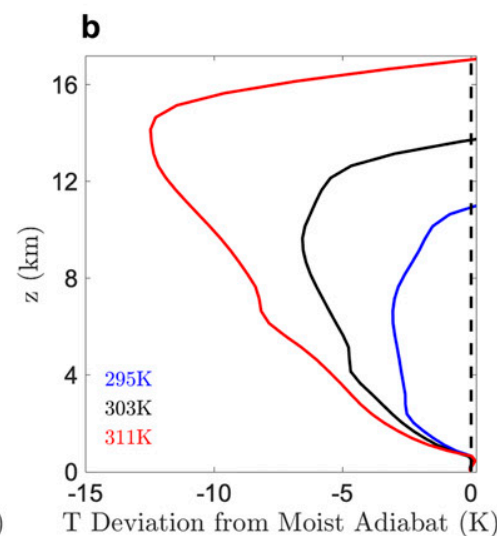

Zero-Buoyancy Plume Model

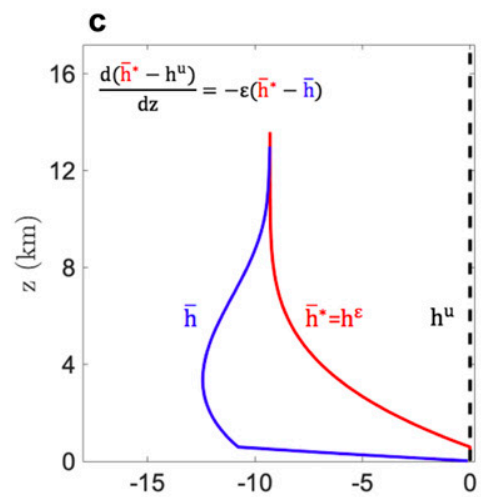

MSE Deviation from Moist Adiabat (K)

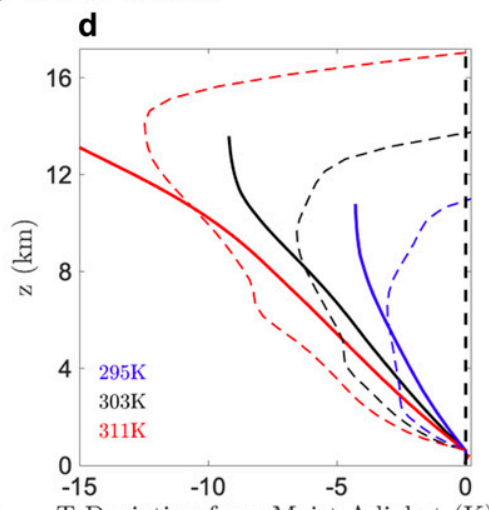

Spectral Plume Model
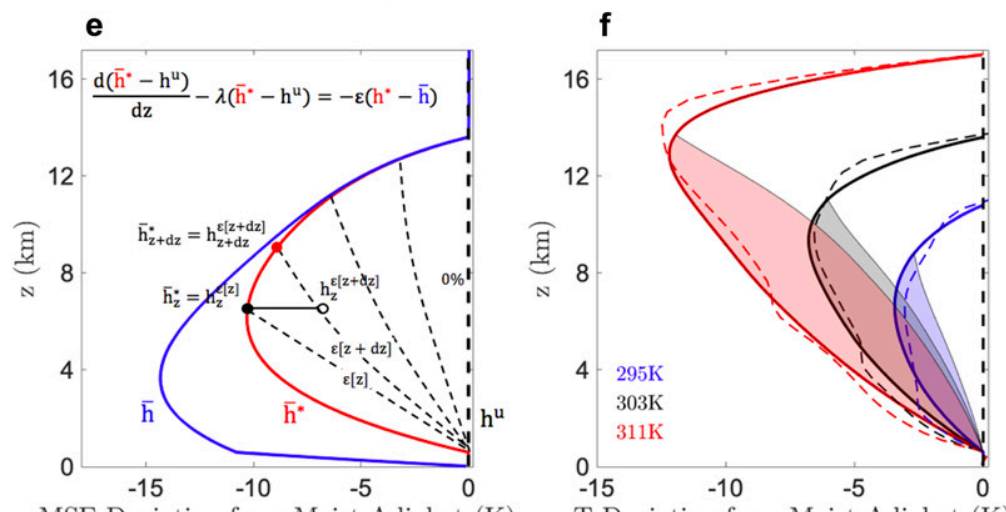

MSE Deviation from Moist Adiabat (K)

$\mathrm{T}$ Deviation from Moist Adiabat (K)

FIG. 2. (left) Vertical profiles of the environmental MSE (blue) and the environmental saturation MSE (red) relative to the undiluted moist adiabat (thick dashed) in the (a) control RCE simulation, (c) the zero-buoyancy plume model, and (e) the spectral plume model. MSE has been normalized by the air heat capacity so that it has a unit of temperature. (right) Vertical profiles of the temperature deviations with various surface temperatures in the (b) RCE simulations, (d) the zero-buoyancy plume model, and (f) the spectral plume model. The color shading in (a) indicates the occurrence likelihood of convective updrafts as a function of height and MSE. RCE simulations are plotted in dashed lines in (d) and (f) for reference. The thin red lines in (e) schematically represent the entraining plumes with distinct entrainment rate. The thin solid lines in (f) show the temperature profiles of the weakly entraining plume with an entrainment rate of $\varepsilon=0.15 \varepsilon_{o}$. The shaded area indicates the integrated temperature difference between this entraining plume and the environment. It represents the magnitude of the integrated buoyancy of this entraining plume. 
changes with warming and its relationship with convective updraft velocities, by building a more realistic conceptual model of RCE. In particular, the bulk plume that is always neutrally buoyant to the environment is replaced by a spectrum of entraining plumes that have distinct entrainment rates and are positively buoyant until they reach neutral buoyancy at different levels of the troposphere. The idea of representing the convective ensemble by a spectrum of entraining plume has been utilized in many subgrid convective schemes (e.g., Arakawa and Schubert 1974; Donner 1993; Wagner and Graf 2010; Chikira and Sugiyama 2010) of GCMs. Convective schemes are used to compute the time-evolving convective heating and moistening tendencies by explicitly considering the interactions of convective clouds with the large-scale environment. Our aim here, however, is to build a conceptual model, which predicts the equilibrated environmental temperature profile given the surface condition, by utilizing the simple entraining picture of convective equilibrium.

The spectral plume model is formulated as follows. Plumes with smaller entrainment rates are assumed to reach their neutral buoyancy at higher levels, such that, for any level $z$ from the cloud base to the tropopause, there is a corresponding plume that reaches neutral buoyancy at this level with an entrainment rate of $\varepsilon[z]$ (schematically shown in Fig. 2e). At its level of neutral buoyancy, the plume MSE $h_{z}^{\varepsilon[z]}$ is equal to the environmental saturation $\operatorname{MSE} \bar{h}_{z}^{*}$ as

$$
\bar{h}_{z}^{*}=h_{z}^{\varepsilon[z]}
$$

The subscript $z$ denotes the level where the MSE is measured and the superscript $\varepsilon[z]$ denotes the specific plume that reaches neutral buoyancy at level $z$ with the corresponding entrainment rate of $\varepsilon[z]$.

The change of the environmental saturation MSE $\bar{h}_{z}^{*}$ from level $z$ to $z+d z$ is then related to the plume MSE as

$$
d \bar{h}^{*}=\bar{h}_{z+d z}^{*}-\bar{h}_{z}^{*}=h_{z+d z}^{\varepsilon[z+d z]}-h_{z}^{\varepsilon[z]},
$$

where $h_{z+d z}^{\varepsilon[z+d z]}$ is the MSE at level $z+d z$ of the plume that reaches neutral buoyancy at level $z+d z$ (with an entrainment rate of $\varepsilon[z+d z])$.

The rhs of (14) can be decomposed as follows (schematically shown in Fig. 2e):

$$
\begin{aligned}
h_{z+d z}^{\varepsilon[z+d z]}-h_{z}^{\varepsilon[z]}= & \left(h_{z+d z}^{\varepsilon[z+d z]}-h_{z}^{\varepsilon[z+d z]}\right) \\
& +\left(h_{z}^{\varepsilon[z+d z]}-h_{z}^{\varepsilon[z]}\right)
\end{aligned}
$$

where $h_{z}^{\varepsilon[z+d z]}$ defines the MSE at level $z$ of the plume that reaches neutral buoyancy at level $[z+d z]$ (with an entrainment rate of $\varepsilon[z+d z])$.

The first term on the rhs of (15) represents the decrease of the plume MSE due to the entrainment. For an infinitesimal $d z$, it is equal to

$$
\begin{aligned}
h_{z+d z}^{\varepsilon[z+d z]}-h_{z}^{\varepsilon[z+d z]} & =-\varepsilon[z+d z]\left(h_{z}^{\varepsilon[z+d z]}-\bar{h}_{z}\right) d z \\
& =-\varepsilon[z]\left(h_{z}^{\varepsilon[z]}-\bar{h}_{z}\right) d z+O^{2}(d z) \\
& =-\varepsilon[z]\left(\bar{h}_{z}^{*}-\bar{h}_{z}\right) d z .
\end{aligned}
$$

The last equality is achieved by neglecting the higherorder terms of $d z$.

The second term on the rhs of (15) represents the MSE increase at level $z$ from the plume with an entrainment rate of $\varepsilon[z]$ to the plume with a smaller entrainment rate of $\varepsilon[z+d z]$ and can be formulated as

$$
h_{z}^{\varepsilon[z+d z]}-h_{z}^{\varepsilon[z]}=\lambda\left(\bar{h}_{z}^{*}-h^{u}\right) d z
$$

with

$$
\lambda \cong \frac{1}{\left\{1+\eta \varepsilon[z]\left(z-z_{b}\right)\right\}} \frac{d \ln \varepsilon}{d z},
$$

and $\eta=0.75$ (see the appendix for detailed derivation). Since plumes with lower entrainment rate are expected to reach neutral buoyancy at higher levels, $d \ln \varepsilon / d z<0$ so $\lambda$ is negative.

Combining (14)-(17) together gives

$$
\frac{d \Delta h}{d z}-\lambda \Delta h=-\varepsilon[z](1-\mathrm{RH}) \frac{L_{v} \overline{q_{v}^{*}}}{C_{p}} .
$$

Equation (19) can be vertically integrated to predict $\Delta h$, and consequently $\Delta T$ according to (8). As shown in Figs. $2 \mathrm{e}$ and $2 \mathrm{f}$, the spectral plume model successfully reproduces the $\mathrm{C}$-shape temperature deviation as well as its increasing magnitude and upward stretch with warming.

Despite being more sophisticated by dropping the zero-buoyancy assumption and considering a spectrum of entraining plumes, the formulation of the spectral plume model, (19), only differs from that of the zerobuoyancy plume model, (11), by a simple term, $\lambda \Delta h$, which accounts for the reduced MSE reduction from entrainment in plumes that reaches higher levels with smaller entrainment rate. The $\lambda \Delta h$ term, however, holds the key for the C-shape profile of the deviation. In the lower troposphere, the entrainment term on the rhs of (19) dominates over the $\lambda \Delta h$ term so that $\Delta h$ becomes more negative with height. In the upper troposphere, the 


\section{RCE Simulations}
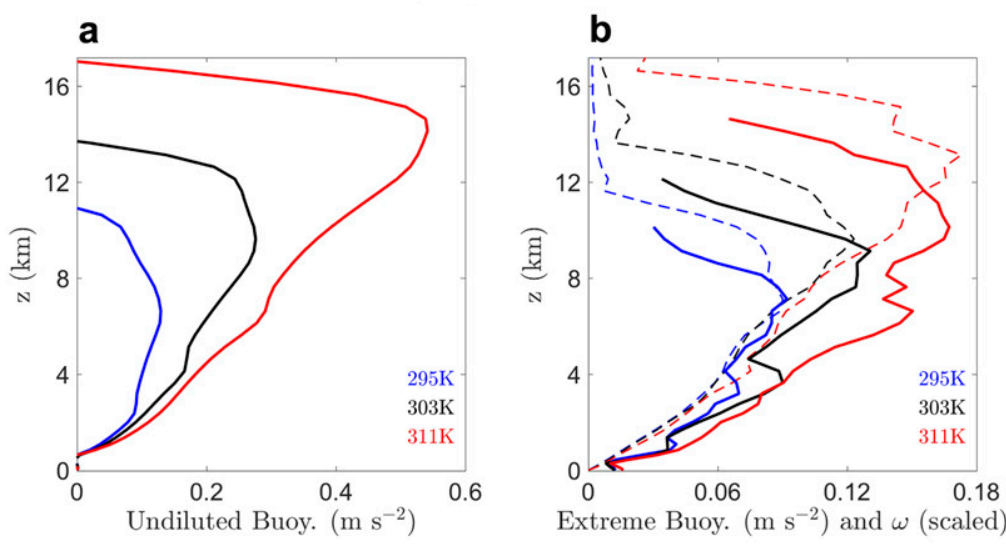

\section{Spectral Plume Model}
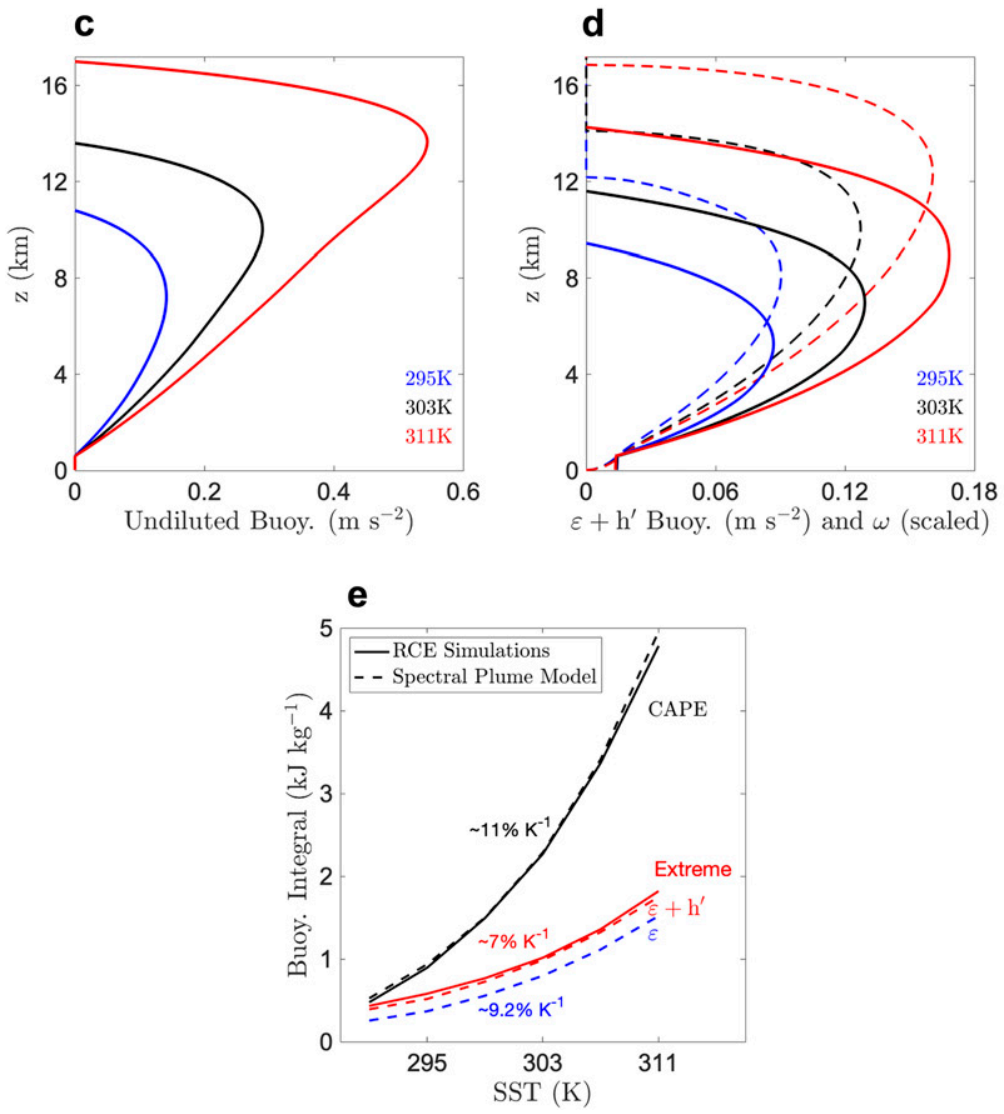

FIG. 3. (a) Buoyancy profiles of the undiluted plumes in RCE simulations with different surface temperatures. (b) Buoyancy (solid) and vertical velocity (dashed) profiles of the extreme convective updraft with a $10^{-5}$ occurrence likelihood in the RCE simulations with different surface temperatures. (c) Buoyancy profiles of the undiluted plumes predicted by the spectral plume model for different surface temperatures. (d) Buoyancy (solid) and vertical velocity (dashed) profiles of the entraining plume with a smaller entrainment rate of $\varepsilon=0.15 \varepsilon_{o}$ and a positive surface MSE anomaly in the spectral plume model for different surface temperatures. (e) CAPE (solid black) and the integrated buoyancy of the extreme convective updraft (solid red) in the RCE simulations as a function of surface temperature. CAPE (dashed black) and the integrated buoyancy of the entraining plume with (dashed red) and without (dashed blue) the positive surface MSE anomaly predicted by the spectral plume model as a function of the surface temperature. 


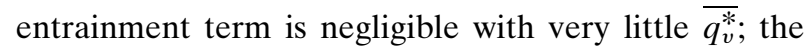
magnitude of $\Delta h$ decreases with height because of the $\lambda \Delta h$ term.

The increasing deviation with warming can now be analytically understood from the spectral plume model, which does not rely on the unrealistic zero-buoyancy assumption. The lhs of (19) is linear in $\Delta h$; the increasing saturation deficit with warming on the rhs simply results in a larger magnitude of $\Delta h$ on the lhs, which consequently leads to a larger temperature deviation $\Delta T$ according to (8). The increase of $\Delta T$ will be more effectively reduced by the increase of the denominator on the lhs of (8) at lower levels because of the larger $\overline{q_{v}^{*}}$ there, leading to amplified temperature response with height. This explains the upward stretch in the relative height of the peak temperature deviation in the troposphere (Fig. 2f).

\section{Change of convective updraft velocities with warming}

Understanding the response of convective updraft velocities to global warming is important for predicting future changes in extreme weather and precipitation (e.g., Baker et al. 1995; Del Genio et al. 2007). Defined as the integrated buoyancy of the undiluted plume, CAPE is often seen as a predictor for convective updraft velocities. In idealized RCE simulations, convective updraft velocities are found to increase correspondingly with CAPE under warming (Romps 2011; Muller et al. 2011), but their fractional increase rate turns out to be smaller than that of CAPE (Singh and O'Gorman 2015). This discrepancy is investigated in Singh and O'Gorman (2015) with a two-plume model in which the environment is assumed to be neutral to a bulk-entraining plume and extreme updrafts are represented by a weakly entraining plume. The two-plume model suggests that the smaller fractional increase of convective velocity compared to that of CAPE is related to the effect of entrainment on both the mean stratification and the updrafts themselves. Here, we seek to provide a clearer understanding by using the spectral plume model, which better reproduces the environmental temperature profile and naturally includes a spectrum of convective updrafts.

The buoyancy profiles of the undiluted plume are shown in Fig. 3a for RCE simulations with various surface temperatures. The undiluted buoyancy is directly related to the temperature deviation (Fig. 2b), so that it increases and stretches upward with warming. The integrated undiluted buoyancy, CAPE, increases with warming by $11 \% \mathrm{~K}^{-1}$ (black solid line in Fig. 3e). Since the spectral plume model accurately predicts the temperature deviation profile (Fig. 2f), it works well to predict the buoyancy profiles (Fig. 3c) and the increasing rate of CAPE with warming (black dashed line in Fig. 3e).

To study the response of the extreme convective updraft to warming, we have categorized convective updrafts in the RCE simulations according to their occurrence likelihood (color shading in Fig. 2a, computed at each level including all points). Figure $3 \mathrm{~b}$ shows the buoyancy and velocity profiles corresponding to the extreme convective updraft that has a $10^{-5}$ occurrence likelihood. Convective updraft velocities are consistently regulated by the associated buoyancies, with maximum velocities consistently located above the peak buoyancy. Consistent with changes in buoyancy profiles, updraft velocities increase with warming, and the increase is most pronounced in the upper troposphere. This indicates limited dynamical impacts on the precipitation (Muller et al. 2011) but substantial influences on the production of lightning and the exchange between the troposphere and the stratosphere (e.g., Sherwood and Dessler 2001; Kuang and Bretherton 2006; Jensen et al. 2007). Consistent with previous studies, we find that the buoyancy and maximum velocity of the extreme convective updraft (Fig. 3b) increase at a smaller fractional rate with warming compared to that of the undiluted plume (Fig. 3a). The integrated buoyancy of the extreme convective updraft only increases with warming by $\sim 7 \% \mathrm{~K}^{-1}$ (red solid line in Fig. 3e), which is significantly smaller than the $11 \%$ increase of CAPE (black solid line in Fig. 3e).

Here, we use the spectral plume model to help understand this smaller fractional increase. We represent the extreme convective updraft as an entraining plume that has a weak entrainment rate of $\varepsilon=0.15 \varepsilon_{o}\left[\varepsilon_{o}\right.$ as defined in (2)] and a positive surface MSE anomaly (chosen to be equal to the standard deviation of the surface MSE). The vertical profile of the plume MSE can be computed according to (10). We can then derive the plume temperature (thin solid lines in Fig. 2f) and buoyancy. The plume updraft velocity $\omega$ is estimated from the plume buoyancy $B$ according to the following equation (Bretherton et al. 2004):

$$
\frac{1}{2} \frac{d \omega^{2}}{d z}=\alpha B-\varepsilon \beta \omega^{2},
$$

where $\alpha=1$ and $\beta=2$. The predicted profiles of the plume buoyancy and updraft velocity are shown in Fig. 3d for various surface temperatures. They show an overall similarity to those in the RCE simulations (Fig. 3b). The most pronounced increase is consistently found in the upper troposphere, as a result of the upward stretch in the buoyancy profile. Furthermore, the spectral plume model successfully reproduces the smaller 
increasing rate of the integrated plume buoyancy (red dashed line in Fig. 3e) compared to that of CAPE.

The slower increase in the plume buoyancy relative to the CAPE can be explained by a combination of the entrainment effect and the positive surface MSE anomaly associated with the extreme convective updraft. Without considering the positive surface MSE anomaly, the entrainment effect alone reduces the increasing rate from $11 \%$ to $9.2 \% \mathrm{~K}^{-1}$ (blue dashed line in Fig. 3e). This reduction is caused by the upward stretch of the temperature deviation profile. The shaded area in Fig. 2f shows the integrated temperature difference between this entraining plume and the environment. As the vertical profile of the environmental temperature deviation stretches upward with warming, the ratio between the plume integrated buoyancy (as measured by the shaded area) and CAPE (as measured by the area between the solid line and the dashed black line) decreases with warming, so that the increasing rate of the integrated plume buoyancy with warming is smaller than that of CAPE. As for the positive surface MSE anomaly, it increases the integrated buoyancy nearly uniformly for the entraining plume under different surface temperatures (from blue dashed line to red dashed line in Fig. 3e). This further reduces the increasing rate of the integrated plume buoyancy with warming from $9.2 \%$ to $7 \% \mathrm{~K}^{-1}$.

\section{Summary}

The tropical atmospheric temperature is typically cooler than that of the moist adiabat, leading to positive CAPE in the tropics. The negative deviation manifests a C-shape profile with the peak deviation in the middle to upper troposphere. This temperature deviation is projected to increase and stretch upward with global warming in both GCMs and idealized RCE simulations, implying important consequences in convective updrafts and extreme weathers.

Previous studies have formulated a zero-buoyancy plume model to understand the increasing magnitude of the temperature deviation with warming. The model proposes an entraining picture of the tropical atmosphere in which the atmospheric temperature deviates from the moist adiabat because of the entrainment effect. It explains that the increasing temperature deviation under global warming is caused by the stronger entrainment dilution of MSE with higher saturation deficit in the free troposphere. The model, however, relies on the unrealistic assumption that clouds are neutrally buoyant and predicts maximum temperature deviation at the tropopause instead of the C-shape profile.
Here, we formulate a more realistic conceptual model of RCE to overcome these limitations and ameliorate the aforementioned entraining picture of the tropical atmosphere. The zero-buoyancy assumption is dropped and the environmental temperature is considered to be controlled by a spectrum of entraining plumes that reach neutral buoyancy at different levels of the troposphere according to their distinct entrainment rate. Given the surface condition and the plume entrainment rate as a function of the neutral buoyancy level, the spectral plume model predicts the environmental temperature profile and properties of the convective updrafts. Despite being more sophisticated by design, the spectral plume model has a concise formulation, which only differs from that of the zero-buoyancy plume model by a simple term that appears because of the plume spectrum. By considering this term, the model reproduces the $\mathrm{C}$-shape deviation and provides a more rigorous explanation for the increasing magnitude and upward stretch of the deviation with warming.

Representing the extreme convective updraft by a weakly entraining plume that is associated with a positive surface MSE anomaly, the model is able to explain the slower increase of extreme convective updraft velocities with warming than that of CAPE. The model indicates that because of the upward stretch in the environmental temperature profile, the ratio between the buoyancy integral of the extreme convective updraft and CAPE decreases so that convective updraft velocities increase at a slower fractional rate compared to that of CAPE. The positive surface MSE anomaly additionally contributes to the reduced increase. We conclude that the spectral plume model presented here provides a useful conceptual model for understanding the tropical temperature profile and convective updraft velocities.

\section{Discussion}

We would like to briefly discuss the parameter sensitivity of this spectral plume model. In particular, we vary the parameter $\varepsilon_{o}$ and $k$ in (2) and the parameter $\eta$ in (18). Increasing $\varepsilon_{o}$ or decreasing $k$ enlarges the predicted temperature deviation as both of them would increase the plume entrainment (Figs. 4a,b). Increasing $\eta$ reduces the parameter $\lambda$ (less negative). Since the parameter $\lambda$ controls the strength of the curve back, this increases the temperature deviation (Fig. 4c). Overall, the qualitative behavior of the spectral plume is found to be robust to these parameters.

It should be noted that the spectral plume model does not explicitly consider the effects of radiation and microphysics. Robe and Emanuel (1996) show that changes in the prescribed radiative cooling could affect the area fraction of convection and may change the updraft velocity 


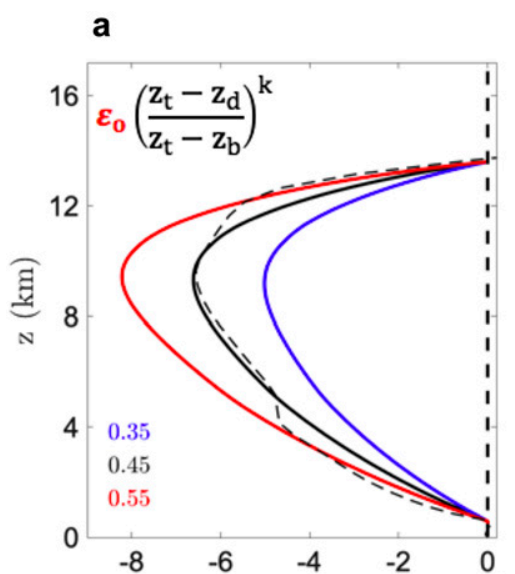

$\mathrm{T}$ Deviation from Moist Adiabat (K) b

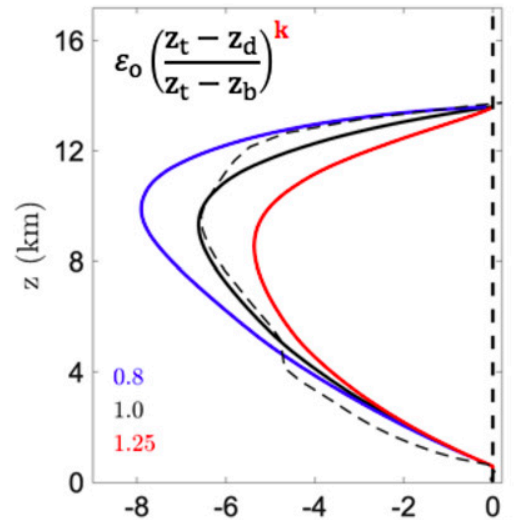

$\mathrm{T}$ Deviation from Moist Adiabat (K)

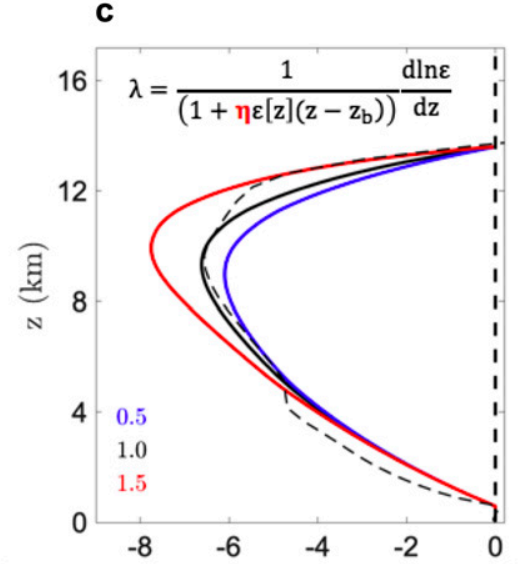

$\mathrm{T}$ Deviation from Moist Adiabat (K)

FIG. 4. Sensitivity of the spectral plume model to (a) $\varepsilon_{o}$, (b) $k$, and (c) $\eta$. The dashed line shows the temperature deviation in the RCE simulation with SST $=303 \mathrm{~K}$ and the color lines show the predictions from the spectral plume model with varying parameters.

at given percentiles. Muller et al. (2011) find that CAPE and updraft velocities respond differently to warming in cases with prescribed and interactive radiation. It might be possible to account for the effect of changing radiative cooling in the spectral plume model by considering their effects on the relationship between the plume entrainment rate and the neutral buoyancy level [(2)], but it is not clear at this point what such effects would be. Furthermore, pseudoadiabatic lift is assumed in this study to simplify the formulation of the spectral plume model. The effect of water loading (Parodi and Emanuel 2009; Singh and O'Gorman 2015) and its changes with warming are thus neglected. Different conclusions have been drawn regarding the effect of precipitation fall speed on updraft velocities (Parodi and Emanuel 2009; Singh and O'Gorman 2015; Pauluis 2016) and future work is desired to further understand this issue.

It is also worthwhile to point out that undiluted plumes are virtually absent in the CM1 RCE simulations. We, however, do include undiluted plumes in the spectral plume model with the consideration that they may exist in reality through organized convections such as mesoscale convective systems and tropical cyclones. We have assumed that the tropopause to be determined by the neutral level of this undiluted plume. This assumption can be easily relaxed. The tropopause can be instead determined by the neutral level of the least-diluted plume so that the predicted temperature profile is cut off at this level and the radiative equilibrium dominates above this level. It is then possible that the radiative process contributes to the upper branch of the observed C-shape profile.

While RCE has been a widely used framework to help understand the tropical atmosphere, it is never really achieved in the tropics because of the existence of Hadley and Walker circulations. In this study, we show that the changes of CAPE and updraft velocities in the tropics can be understood to first order without considering these large-scale circulations. It would be an interesting avenue for future work to investigate their impacts.

Acknowledgments. We are grateful to George Bryan for providing the CM1 model and to Martin Singh for providing the MATLAB code of the zero-buoyancy plume model for use by the community. This work is supported by the National Science Foundation (NSF-1637450). Numerical simulations are conducted using the computing resources provided by NCAR Cheyenne: HPE/SGI ICE XA System (University Community Computing; https://doi.org/10.5065/D6RX99HX). We thank Martin Singh, Tristan H. Abbott, and other anonymous reviewer(s) for their contribution to the peer review of this work.

\section{APPENDIX}

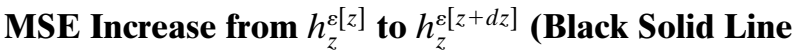 in Fig. 2e) due to Reduced Entrainment}

For the plume with the entrainment rate $\varepsilon[z]$, its MSE, $h^{\varepsilon[z]}$, decreases monotonically with the height because of the entrainment from the environment; that is,

$$
\frac{d h^{\varepsilon[z]}}{d z}=-\varepsilon[z]\left(h^{\varepsilon[z]}-\bar{h}\right) .
$$

Integrating (A1) from the cloud base to the level $z$ gives

$$
h_{z}^{\varepsilon[z]}-h^{u}=\int_{z_{b}}^{z}-\varepsilon[z]\left(h_{z^{\prime}}^{\varepsilon[z]}-\bar{h}\right) d z^{\prime} .
$$

Similarly, for the plume with the entrainment rate $\varepsilon[z+d z]$, one has 
a

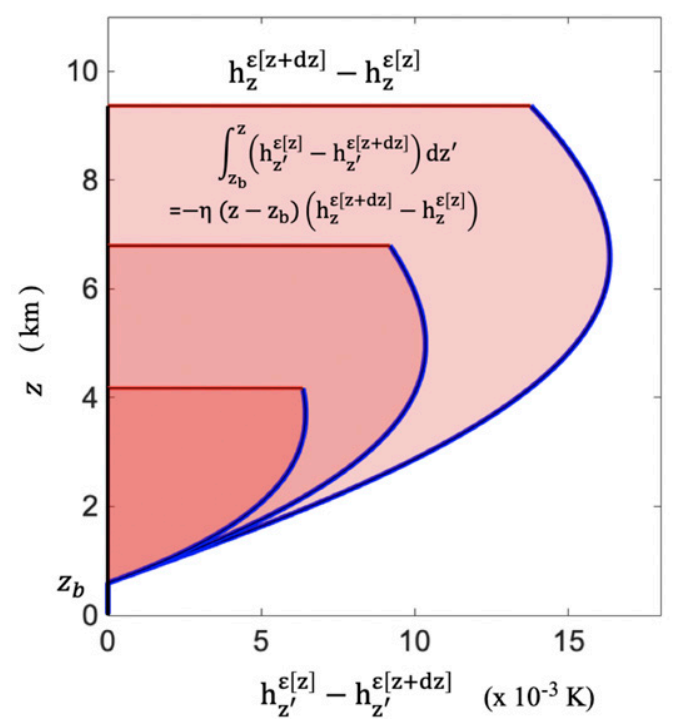

b

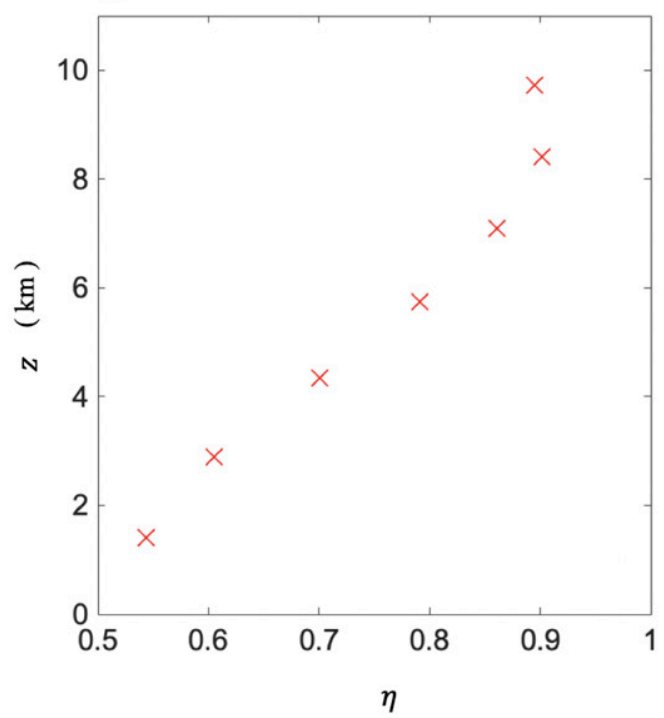

FIG. A1. (a) The vertical profile of $h_{z^{\prime}}^{\varepsilon[z]}-h_{z^{\prime}}^{\varepsilon[z+d z]}$ as a function of $z^{\prime}$ for three different levels $z$ computed with $\eta$ initially specified as a constant value of 0.75 . (b) Plot of $\eta$ at different levels $z$ is estimated as the shaded area in (a) divided by both the length of $h_{z}^{\varepsilon[z]}-h_{z}^{\varepsilon[z+d z]}$ and the width of $z-z_{b}$.

$$
h_{z}^{\varepsilon[z+d z]}-h^{u}=\int_{z_{b}}^{z}-\varepsilon[z+d z]\left(h_{z^{\prime}}^{\varepsilon[z+d z]}-\bar{h}\right) d z^{\prime}
$$

Subtracting (A2) from (A3) leads to

$$
\begin{aligned}
h_{z}^{\varepsilon[z+d z]}-h_{z}^{\varepsilon[z]}= & \int_{z_{b}}^{z}-(\varepsilon[z+d z]-\varepsilon[z])\left(h_{z^{\prime}}^{\varepsilon[z]}-\bar{h}\right) d z^{\prime} \\
& +\int_{z_{b}}^{z} \varepsilon[z+d z]\left(h_{z^{\prime}}^{\varepsilon[z]}-h_{z^{\prime}}^{\varepsilon[z+d z]}\right) d z^{\prime}
\end{aligned}
$$

The first integrand on the rhs of (A4) can be written as

$$
\begin{aligned}
\int_{z_{b}}^{z} & -(\varepsilon[z+d z]-\varepsilon[z])\left(h_{z^{\prime}}^{\varepsilon[z]}-\bar{h}\right) d z^{\prime} \\
& =\frac{\varepsilon[z+d z]-\varepsilon[z]}{\varepsilon[z]} \int_{z_{b}}^{z}-\varepsilon[z]\left(h_{z^{\prime}}^{\varepsilon[z]}-\bar{h}\right) d z^{\prime} \\
& =\frac{d \varepsilon[z]}{\varepsilon[z]}\left(h_{z}^{\varepsilon[z]}-h^{u}\right)=\frac{d \varepsilon[z]}{\varepsilon[z]}\left(\bar{h}_{z}^{*}-h^{u}\right) .
\end{aligned}
$$

The second integrand at the rhs of (A4) can be written as

$$
\begin{aligned}
& \int_{z_{b}}^{z} \varepsilon[z+d z]\left(h_{z^{\prime}}^{\varepsilon[z]}-h_{z^{\prime}}^{\varepsilon[z+d z]}\right) d z^{\prime} \\
& \quad=\varepsilon[z] \int_{z_{b}}^{z}\left(h_{z^{\prime}}^{\varepsilon[z]}-h_{z^{\prime}}^{\varepsilon[z+d z]}\right) d z^{\prime} \\
& \quad \cong-\eta \varepsilon[z]\left(z-z_{b}\right)\left(h_{z}^{\varepsilon[z+d z]}-h_{z}^{\varepsilon[z]}\right) .
\end{aligned}
$$

The first equality is achieved with an infinitesimal $d z$ (we use $d z=20 \mathrm{~m}$ in our computation). The second equality is achieved by introducing the parameter $\eta$. The exact value of $\eta$ depends on the vertical profile of $h_{z^{\prime}}^{\varepsilon[z]}-h_{z^{\prime}}^{\varepsilon[z+d z]}$ as a function of $z^{\prime}$. For example, if $h_{z^{\prime}}^{\varepsilon[z]}-h_{z^{\prime}}^{\varepsilon\left[z^{z^{\prime}} d z\right]}$ increases linearly with $z^{\prime}$ from $z_{b}$ to $z, \eta$ will be 0.5 . Figure A1 shows the vertical profile of $h_{z^{\prime}}^{\varepsilon[z]}-h_{z^{\prime}}^{\varepsilon[z+d z]}$ computed from the spectral-plume model with $\eta$ initially specified as 0.75 . The resulting $h_{z^{\prime}}^{\varepsilon[z]}-h_{z^{\prime}}^{\varepsilon[z+d z]}$ turns out to increase nonlinearly with $z^{\prime}$, and the value of $\eta$ changes with $z$. To keep the simplicity of our conceptual model, we however use a constant $\eta=0.75$ that roughly estimates the mean value across the troposphere. The qualitative behavior of the spectral plume model is rather robust to the exact value of $\eta$, as shown in Fig. 4c.

Substituting (A5) and (A6) into (A4) gives

$$
\begin{aligned}
h_{z}^{\varepsilon[z+d z]}-h_{z}^{\varepsilon[z]}= & \frac{d \varepsilon[z]}{\varepsilon[z]}\left(\bar{h}_{z}^{*}-h^{u}\right) \\
& -\eta \varepsilon[z]\left(z-z_{b}\right)\left(h_{z}^{\varepsilon[z+d z]}-h_{z}^{\varepsilon[z]}\right) .
\end{aligned}
$$

Then $h_{z}^{\varepsilon[z+d z]}-h_{z}^{\varepsilon[z]}$ can be written as

$$
h_{z}^{\varepsilon[z+d z]}-h_{z}^{\varepsilon[z]}=\lambda\left(\bar{h}_{z}^{*}-h^{u}\right) d z
$$

where

$$
\lambda=\frac{1}{\left\{1+\eta \varepsilon[z]\left(z-z_{b}\right)\right\}} \frac{d \ln \varepsilon}{d z}
$$




\section{REFERENCES}

Arakawa, A., and W. H. Schubert, 1974: Interaction of a cumulus cloud ensemble with the large-scale environment, part I. J. Atmos. Sci., 31, 674-701, https://doi.org/10.1175/1520-0469(1974) 031<0674:IOACCE > 2.0.CO;2.

Baker, M. B., H. J. Christian, and J. Latham, 1995: A computational study of the relationships linking lightning frequency and other thundercloud parameters. Quart. J. Roy. Meteor. Soc., 121, 1525-1548, https://doi.org/10.1002/qj.49712152703.

Bretherton, C. S., J. R. McCaa, and H. Grenier, 2004: A new parameterization for shallow cumulus convection and its application to marine subtropical cloud-topped boundary layers. Part I: Description and 1D results. Mon. Wea. Rev., 132, 864-882, https://doi.org/10.1175/1520-0493(2004) $132<0864$ :ANPFSC $>2.0 . \mathrm{CO} ; 2$.

_ , P. N. Blossey, and M. Khairoutdinov, 2005: An energybalance analysis of deep convective self-aggregation above uniform SST. J. Atmos. Sci., 62, 4273-4292, https://doi.org/ 10.1175/JAS3614.1.

Brooks, H., 2013: Severe thunderstorms and climate change. Atmos. Res., 123, 129-138, https://doi.org/10.1016/j.atmosres.2012.04.002.

Bryan, G. H., and J. M. Fritsch, 2002: A benchmark simulation for moist nonhydrostatic numerical models. Mon. Wea. Rev., 130, 2917-2928, https://doi.org/10.1175/1520-0493(2002)130<2917: ABSFMN $>2.0 . C O ; 2$.

Chikira, M., and M. Sugiyama, 2010: A cumulus parameterization with state-dependent entrainment rate. Part I: Description and sensitivity to temperature and humidity profiles. J. Atmos. Sci., 67, 2171-2193, https://doi.org/10.1175/2010JAS3316.1.

Del Genio, A. D., M. S. Yao, and J. Jonas, 2007: Will moist convection be stronger in a warmer climate? Geophys. Res. Lett., 34, L16703, https://doi.org/10.1029/2007GL030525.

Donner, L. J., 1993: A cumulus parameterization including mass fluxes, vertical momentum dynamics, and mesoscale effects. J. Atmos. Sci., 50, 889-906, https://doi.org/10.1175/1520-0469(1993) 050<0889:ACPIMF $>2.0$. CO;2.

Dunion, J. P., 2011: Rewriting the climatology of the tropical North Atlantic and Caribbean Sea atmosphere. J. Climate, 24, 893-908, https://doi.org/10.1175/2010JCLI3496.1.

Fasullo, J., 2012: A mechanism for land-ocean contrasts in global monsoon trends in a warming climate. Climate Dyn., 39, 1137-1147, https://doi.org/10.1007/s00382-011-1270-3.

Gent, P. R., and Coauthors, 2011: The Community Climate System Model version 4. J. Climate, 24, 4973-4991, https://doi.org/ 10.1175/2011JCLI4083.1.

Held, I. M., 1982: On the height of the tropopause and the static stability of the troposphere. J. Atmos. Sci., 39, 412-417, https:// doi.org/10.1175/1520-0469(1982)039<0412:OTHOTT>2.0.CO;2.

__ R. S. Hemler, and V. Ramaswamy, 1993: Radiativeconvective equilibrium with explicit two-dimensional moist convection. J. Atmos. Sci., 50, 3909-3927, https://doi.org/ 10.1175/1520-0469(1993)050<3909:RCEWET>2.0.CO;2.

Jensen, E. J., A. S. Ackerman, and J. A. Smith, 2007: Can overshooting convection dehydrate the tropical tropopause layer? J. Geophys. Res., 112, D11209, https://doi.org/10.1029/2006JD007943.

Jordan, C. L., 1958: Mean soundings for the West Indies area. J. Meteor., 15, 91-97, https://doi.org/10.1175/1520-0469(1958) 015<0091:MSFTWI>2.0.CO;2.

Kuang, Z., and C. S. Bretherton, 2006: A mass-flux scheme view of a high-resolution simulation of a transition from shallow to deep cumulus convection. J. Atmos. Sci., 63, 1895-1909, https://doi.org/10.1175/JAS3723.1.
Lepore, C., D. Veneziano, and A. Molini, 2015: Temperature and CAPE dependence of rainfall extremes in the eastern United States. Geophys. Res. Lett., 42, 74-83, https://doi.org/10.1002/ 2014 GL062247.

Lin, C., and A. Arakawa, 1997: The macroscopic entrainment processes of simulated cumulus ensemble. Part I: Entrainment sources. J. Atmos. Sci., 54, 1027-1043, https://doi.org/10.1175/ 1520-0469(1997)054<1027:TMEPOS > 2.0.CO;2.

Lin, Y.-L., R. D. Farley, and H. D. Orville, 1983: Bulk parameterization of the snow field in a cloud model. J. Climate Appl. Meteor., 22, 1065-1092, https://doi.org/10.1175/1520-0450(1983) $022<1065$ :BPOTSF $>2.0 . \mathrm{CO} ; 2$.

Manabe, S., and R. T. Wetherald, 1967: Thermal equilibrium of the atmosphere with a given distribution of relative humidity. J. Atmos. Sci., 24, 241-259, https://doi.org/10.1175/1520-0469(1967) $024<0241$ :TEOTAW $>2.0 . \mathrm{CO} ; 2$.

Muller, C. J., P. A. O'Gorman, and L. E. Back, 2011: Intensification of precipitation extremes with warming in a cloud-resolving model. J. Climate, 24, 2784-2800, https://doi.org/10.1175/ 2011JCLI3876.1.

Parodi, A., and K. A. Emanuel, 2009: A theory for buoyancy and velocity scales in deep moist convection. J. Atmos. Sci., 66, 3449-3463, https://doi.org/10.1175/2009JAS3103.1.

Pauluis, O. M., 2016: The mean air flow as Lagrangian dynamics approximation and its application to moist convection. J. Atmos. Sci., 73, 4407-4425, https://doi.org/10.1175/JAS-D-15-0284.1.

, and A. A. Mrowiec, 2013: Isentropic analysis of convective motions. J. Atmos. Sci., 70, 3673-3688, https://doi.org/10.1175/ JAS-D-12-0205.1.

Riehl, H., and J. S. Malkus, 1958: On the heat balance in the equatorial trough zone. Geophysica, 6, 503-538.

Robe, F. R., and K. A. Emanuel, 1996: Moist convective scaling: Some inferences from three-dimensional cloud ensemble simulations. J. Atmos. Sci., 53, 3265-3275, https://doi.org/10.1175/ 1520-0469(1996)053<3265:MCSSIF >2.0.CO;2.

Romps, D. M., 2011: Response of tropical precipitation to global warming. J. Atmos. Sci., 68, 123-138, https://doi.org/10.1175/ 2010JAS3542.1.

_ , and Z. Kuang, 2010a: Nature versus nurture in shallow convection. J. Atmos. Sci., 67, 1655-1666, https://doi.org/10.1175/ 2009JAS3307.1.

$\longrightarrow$, and - 2010b: Do undiluted convective plumes exist in the upper tropical troposphere? J. Atmos. Sci., 67, 468-484, https:// doi.org/10.1175/2009JAS3184.1.

, and R. Öktem, 2015: Stereo photogrammetry reveals substantial drag on cloud thermals. Geophys. Res. Lett., 42, 5051-5057, https://doi.org/10.1002/2015GL064009.

- J. T. Seeley, D. Vollaro, and J. Molinari, 2014: Projected increase in lightning strikes in the United States due to global warming. Science, 346, 851-854, https://doi.org/ 10.1126/science.1259100.

Seeley, J. T., and D. M. Romps, 2015: Why does tropical convective available potential energy (CAPE) increase with warming? Geophys. Res. Lett., 42, 10 429-10 437, https://doi.org/10.1002/ 2015GL066199.

Sherwood, S. C., and A. E. Dessler, 2001: A model for transport across the tropical tropopause. J. Atmos. Sci., 58, 765-779, https://doi.org/10.1175/1520-0469(2001)058<0765: AMFTAT $>2.0 . \mathrm{CO} ; 2$

Singh, M. S., and P. A. O'Gorman, 2013: Influence of entrainment on the thermal stratification in simulations of radiative-convective equilibrium. Geophys. Res. Lett., 40, 4398-4403, https://doi.org/10.1002/grl.50796. 
and - 2015: Increases in moist-convective updraft velocities with warming in radiative-convective equilibrium. Quart. J. Roy. Meteor. Soc., 141, 2828-2838, https://doi.org/ 10.1002/qj.2567.

Z. Kuang, E. D. Maloney, W. M. Hannah, and B. O. Wolding, 2017: Increasing potential for intense tropical and subtropical thunderstorms under global warming. Proc. Natl. Acad. Sci. USA, 114, 11 657-11 662, https://doi.org/10.1073/ pnas. 1707603114.

Sobel, A. H., and S. J. Camargo, 2011: Projected future changes in tropical summer climate. J. Climate, 24, 473-487, https:// doi.org/10.1175/2010JCLI3748.1.

Stommel, H., 1947: Entrainment of air into a cumulus cloud. J. Meteor., 4, 91-94, https://doi.org/10.1175/1520-0469(1947) $004<0091$ :EOAIAC $>2.0 . \mathrm{CO} ; 2$.

Taylor, K. E., R. J. Stouffer, and G. A. Meehl, 2012: An overview of CMIP5 and the experiment design. Bull. Amer. Meteor. Soc., 93, 485-498, https://doi.org/10.1175/BAMS-D-11-00094.1.
Taylor, T. R., and M. B. Baker, 1991: Entrainment and detrainment in cumulus clouds. J. Atmos. Sci., 48, 112-121, https://doi.org/ 10.1175/1520-0469(1991)048<0112:EADICC >2.0.CO;2.

Tompkins, A. M., and G. C. Craig, 1998: Radiative-convective equilibrium in a three-dimensional cloud-ensemble model. Quart. J. Roy. Meteor. Soc., 124, 2073-2097, https://doi.org/ 10.1256/SMSQJ.55012.

Wagner, T. M., and H. F. Graf, 2010: An ensemble cumulus convection parameterization with explicit cloud treatment. J. Atmos. Sci., 67, 3854-3869, https://doi.org/10.1175/ 2010JAS3485.1.

Williams, E. R., S. Rutledge, S. Geotis, N. Renno, E. Rasmussen, and T. Rickenbach, 1992: A radar and electrical study of tropical "hot towers." J. Atmos. Sci., 49, 1386-1395, https:// doi.org/10.1175/1520-0469(1992)049<1386:ARAESO>2.0.CO;2.

$\mathrm{Xu}, \mathrm{K} .-\mathrm{M}$., and K. A. Emanuel, 1989: Is the tropical atmosphere conditionally unstable? Mon. Wea. Rev., 117, 1471-1479, https:// doi.org/10.1175/1520-0493(1989)117<1471:ITTACU>2.0.CO;2. 